\title{
Study of Water Management Development in Petung Swamp Areas at the Province of East Kalimantan
}

\author{
Agus Adi Pramono ${ }^{1}$, Suhardjono ${ }^{1}$, Moch. Sholichin ${ }^{1}$ \\ ${ }^{1}$ Water Resources Engineering Department, Universitas Brawijaya, Malang, 65145, \\ Indonesia \\ agusadi001@gmail.com
}

Received 28-06-2021; accepted 09-08-2021

\begin{abstract}
Swamp area usually seen at the debouchment that close to the sea. Swamp area can be used for farming area if it has a proper water management system. However, water management at the swamp area is hard to do and can be potentially become over drained in some areas. The aim of this study is to locate the potential areas that are able to occur over drained at Petung Swamp Irrigation System and able to find solutions of the problems. The first step to do is doing survey to know the condition of the channel, finding hydrological data, and tidal data. The hydrological data are intended to get modulus drain discharge. The next step is running data with HecRAS application to get the result of water channel profile of all channels. After that, analyzing the groundwater level could be done and meet the conclusion whether the channel is having over drained or not. The result of this study is the areas of Petung Swamp Irrigation that mostly having over drained condition are at the headwaters because the tide cannot reach the upper areas. The areas also have no floodgates, so the water journey couldn't be occurred and not evenly distributed.
\end{abstract}

Keywords: groundwater level, over drained, swamp area, tides

\section{Introductions}

The increasingly limited productive land is one of the problems faced by the government in achieving the national food security program. With a lot of land conversion from technically irrigated land to nonagricultural land, it is also a factor in reducing agricultural crop production [1].

In the other hand, swamp areas are one of the nature wealth that is able to functioned that it can become one of the sources of growth that is considered capable of encouraging the pace of economic development and the prosperity of its people [2].

The main problem of the less significant development of swamp areas into agricultural is the quality and the amount of the water availability on those swamp lands. In addition, the poor condition of drainage system also capable to cause toxic substance which are harmful to the roots and causing

Cite this as: Pramono, A.A., Suhardjono \& Sholichin, M. (2021). Study of Water Management Development in Petung Swamp Areas at the Province of East Kalimantan. Civil and Environmental Science Journal (Civense), 4(2), 173-182. doi: https://doi.org/10.21776/ub.civense.2021.00402.7 
agricultural failure on the swamp. Thus, or the success of agriculture in swamps is water management at the micro and macro levels in swamps [3].

The development of agricultural land in tidal swamp areas must pay attention to all supporting aspects in order to be successful. In tidal swamp land, agricultural land is easily contaminated by heavy metals due to stagnant wastewater. The presence of heavy metals in tidal water must get serious attention because it has serious impacts, including: the potential for carcinogens and metals have conservative properties that tend to accumulate in the human body $[4,10]$.

In order to avoid the wastewater stagnant, draining the water on the swamps area is the most important thing to do, therefore the land is suitable for cultivation. However, on its process is potentially happen the over drained issues.

Over drained condition for a long time can cause peatlands to become dry and unable to absorb water again, because they experience irreversible drying conditions. This very dry condition also has the potential to cause land fires [5].

On this study, the analysis is done to figuring out the swamp land condition of Petung Swamp Irrigation whether experiencing the over drained condition or not by doing the analyzation with the help of HecRAS application that is useful to knowing the water level of the canal and then the groundwater level will be known. After knowing the status, the aim of this study is to finding some solutions and recommendations for the problems before it gets harder to handle. Furthermore, the solutions that given on this study are to make the water system management better so it can help the government program of achieving the national food security.

The optimum groundwater level to avoid the dryness and land fire is about $60-100 \mathrm{~cm}$. For the effort to maintain the the stability of the groundwater level remains shallow $( \pm<70 \mathrm{~cm})$, it is necessary to install water gates [6].

\section{Materials and Methods}

This study was conducted by conducting an analysis to obtain the groundwater level on the land so that it can determine whether the land is experiencing over drain or not. In the analysis, the HecRAS application is used to assist in modeling so that the water level of the canal is obtained which will further be used as one of the parameters in calculating the groundwater level using the modified Ellips Kirkham method.

In the analysis to get the groundwater level on the land, soil conductivity data is also needed obtained from soil drilling in the field at several points.

\subsection{Land Hydrotopographic}

Hydrotopographic is a condition that shown the water level to the topography around it.

The type of land hydro topography at the swamp areas affect the type of water system used in order to optimize the performance of the existing channel [7].

The hydrotopographic category is an indication of the extent to which the possibility of overflowing water can inundate the land, and conversely the existing inundation can be drained. Based on the category, tidal swamp land is divided into 4 categories, namely categories A, B, C, and D. Category A is swamp land that is flooded by tidal water during the dry and rainy seasons. Category B is swamp land that is overflowed by tidal water only during the rainy season. Category $\mathrm{C}$ is land that is not flooded with high tide all the time. While category $\mathrm{D}$ is swamp land that is not at all flooded by tides.

\subsection{Ellips Kirkham Model Modification}

One of the ways that can be used in determining the groundwater level is to use a modified method of the Kirkham ellipse model [8].

In the research conducted by Ngudiantoro, it is slightly different from that conducted by Kirkham. In Ngudiantoro's research, the modeling is done more simply, with the following assumptions:

(1) Elliptical model for groundwater table introduces the concept of mirror image,

(2) The depth of the channel to the impermeable layer, and 
(3) The slope of the land surface is relatively small.

The equations used in the modification method of the Kirkham ellipse model are as follows:

$h^{2}(x)=h_{w}^{2}+\frac{h_{w}}{s+\ell} \frac{R-E T}{K}\left(2 s x-x^{2}\right)$

With:

$\mathrm{h}(\mathrm{x})=$ Groundwater level above the impermeable layer at a distance $\mathrm{x}$ from the channel (m);

hw = water level in the channel above the impermeable layer $(\mathrm{m})$;

$\mathrm{R} \quad=$ Rainfall $(\mathrm{mm} / \mathrm{day})$;

ET = Evapotranspiration ( $\mathrm{mm} /$ day);

$\mathrm{K}=$ Soil hydraulic conductivity $(\mathrm{mm} /$ day);

$\mathrm{x} \quad=$ Distance from channel $(\mathrm{m})$;

$2 \mathrm{~s} \quad=$ Distance between channels $(\mathrm{m})$;

$1=$ Channel width $(\mathrm{m})$.

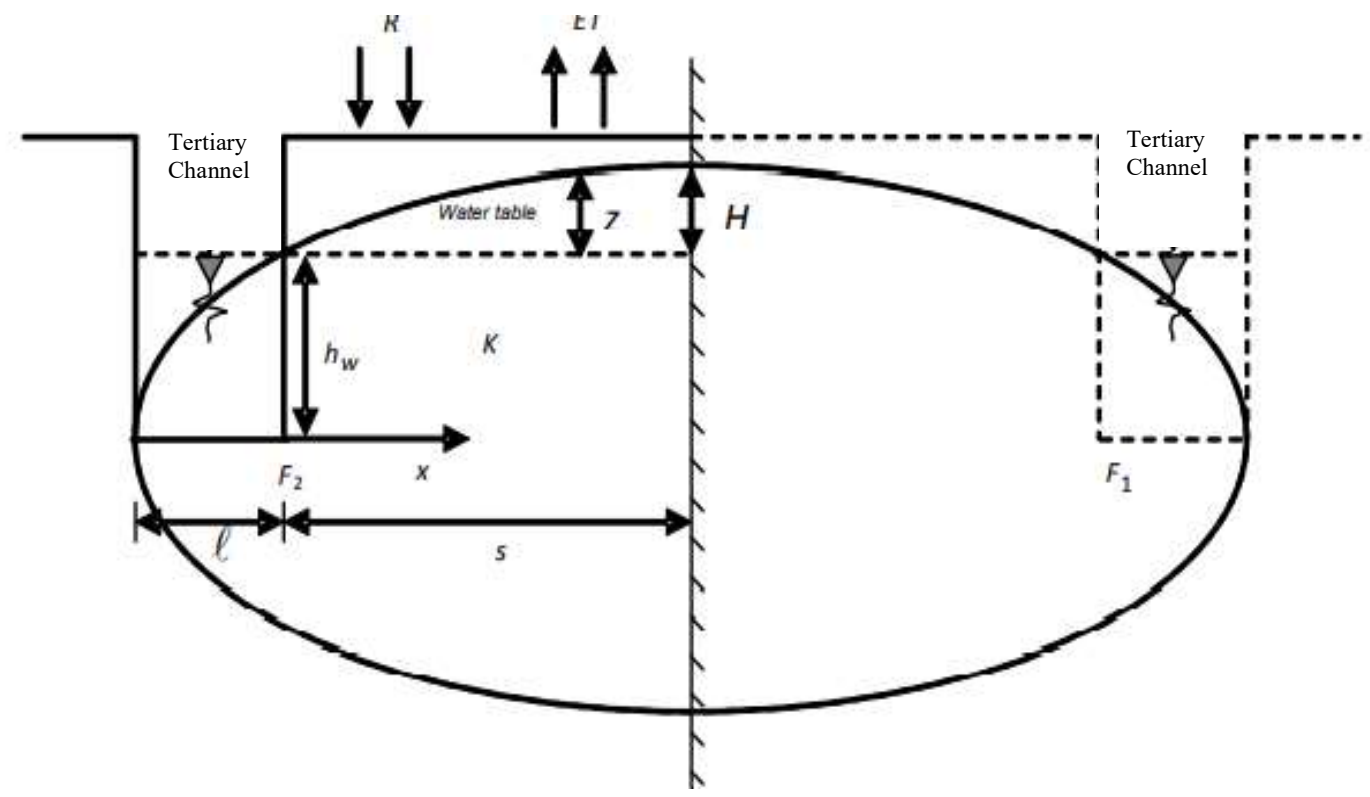

Figure 1. The Illustration of The Modified Ellips Kirkham Model

The figure above is explaining the illustration for the calculation of finding the groundwater level between 2 channels. The illustration above is explaining the assumpsion of the modified model of Ellips Kirkham formula. The modified formula looks simpler than the actual formula. The calculation takes approximately in the middle between 2 channels, so it can make the calculation easier.

\subsection{Over Drained}

Over-draining is a condition where the disposal of excess water in swamps occurs excessively so that the groundwater level decreases [11].

The occurrence of over-drained in the long term can cause dry conditions do not return. Irreversible drying is a peat condition with a moisture content of $<100 \%$. In this condition, peat no longer has the ability to absorb water and nutrients, so it can no longer be planted. In this condition, peat will easily burn and be carried away by water [9].

Some ways that can be done to reduce the risk of over draining are as follows:

1. Adjust the distance between the drainage channels.

2. Operate the sluice gate in the channel, in order to maintain the groundwater level and the pyrite layer will always be in anaerobic condition.

3. Making long storage at the upstream of the channel. 
4. Conduct water management on peatlands.

\section{Result and Discussion}

Hydrotopographic analysis needs to be carried out at the beginning of the analysis to determine whether the Petung swamp land is included in the radish swamp or tidal swamp land. If the Petung Swamp Irrigation System falls into the category of lowland swamp land, then the next calculation only uses rainfall. Meanwhile, if the Petung swamp irrigation area is included in the tidal swamp category, then the tidal height of sea water is one of the parameters included in the next calculation.

In determining the hydrotopographic category, it is necessary to analyze the land surface elevation in the field and the highest tide level. The highest tide height obtained from the survey results for 15 days was $2.29 \mathrm{~m}$.

\subsection{Figure}

From the analysis carried out, in Figure 2, it can be seen that the study location, namely the Petung swamp irrigation area Main Channel I and Main Canal II, is included in the overflow type C (Green shading) covering an area of 459.4 ha which is land still affected by tides the sea and overflow type D (blue shading) covering an area of 87.26 ha which is land without any influence from tides and has the potential to be over drained. For further, it is necessary to do an analysis using Hecras to find out how far the influence of tides on the channel in Main Channel I and II Petung Swamp Irrigation System.

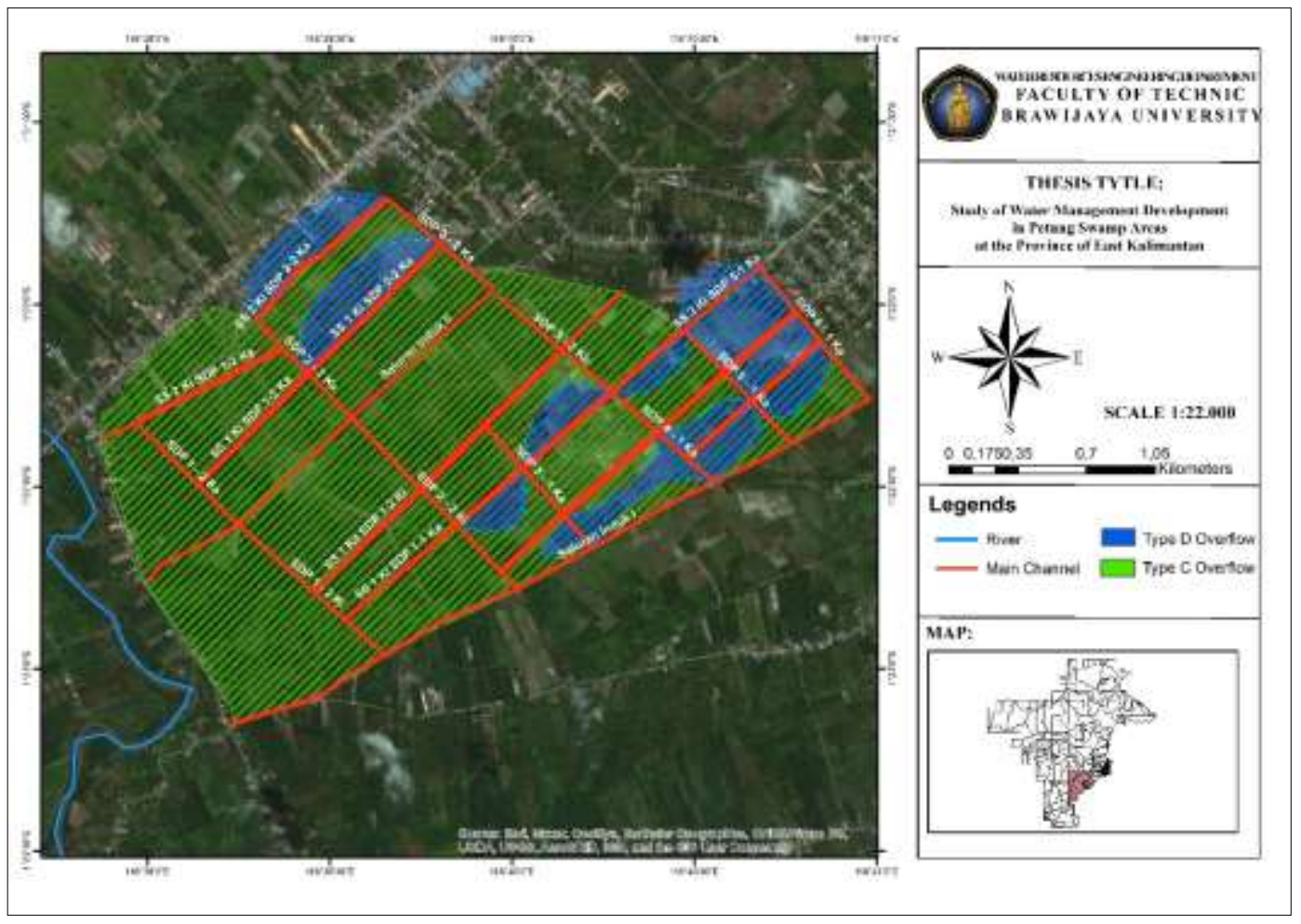

Figure 2. Petung Swamp Irrigation Area Hydrotopographic Map

Hec-Ras analysis was carried out for each stake in the field. The results obtained from the analysis using the Hec-Ras application are in the form of a channel water level profile (Figures 3 and 4). 


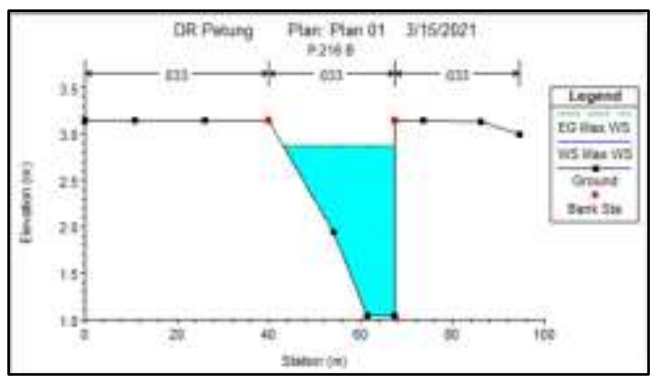

Figure 3. Example of Channel Water Level Profile Results in Hec-Ras Modeling (Main Channel I).

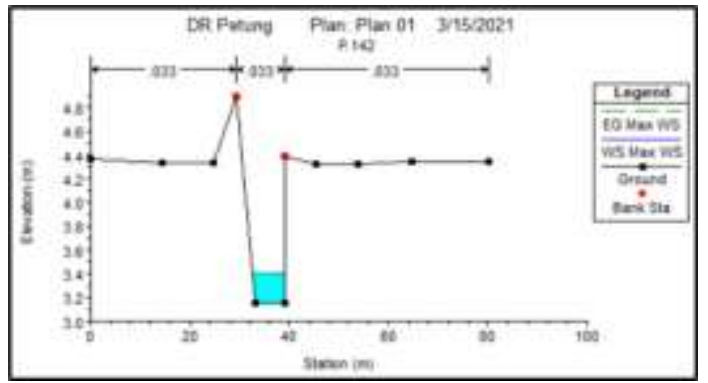

Figure 4. Example of Channel Water Level Profile Results in Hec-Ras Modeling (Main Channel II).

From the figures above, the cross section in some stakes are showed that water fills the channel, like in the stakes P.216 B from main channel I. Therefore, the cross section in some stakes are showed have less water, like in the stake P.142 from main channel II.

\subsection{Table}

In the groundwater table profile analysis, all the stakes that have been made in the field are inputted and analyzed in the HecRAS application.

The results of the hecRAS modeling will be one of the parameters in the calculation of the groundwater level using the modified Kirkham elliptical model along with other parameters, namely evapotranspiration, effective rainfall, and soil conductivity.

The following is a recapitulation of the height of the canal water level profile at all stake points.

Table 1. Channel Water Level Profile Recapitulation

$\begin{array}{lll}\text { No Channel } & \text { Stake Point } & \text { hw (m) }\end{array}$

$1 \quad$ S Drain Induk $1 \quad$ P.216 B 1.83

P.243 B 


\begin{tabular}{|c|c|c|c|}
\hline No & Channel & Stake Point & hw (m) \\
\hline \multirow{8}{*}{2} & \multirow{8}{*}{$\begin{array}{l}\text { Main } \\
\text { Channel } 2\end{array}$} & P.261 & 1.76 \\
\hline & & P. 92 B & 1.73 \\
\hline & & P.131 B Right & 1.64 \\
\hline & & P.131 B Left & 1.64 \\
\hline & & P. 25 B Right & 1.85 \\
\hline & & P.25 B Left & 1.85 \\
\hline & & P. 11 A Right & 1.29 \\
\hline & & P. 11 A Left & 1.29 \\
\hline \multirow[t]{2}{*}{3} & \multirow{2}{*}{$\begin{array}{l}\text { SS1Ki SDP } \\
1-2 \mathrm{Ka}\end{array}$} & P.325 A Left & 1.24 \\
\hline & & P.325 A Right & 1.24 \\
\hline \multirow[t]{2}{*}{4} & $\begin{array}{l}\text { SS2Ki SDP } \\
1-2 \mathrm{Ka}\end{array}$ & CP. 14 & 1.38 \\
\hline & SDP $1-2 \mathrm{Ka}$ & & \\
\hline \multirow[t]{3}{*}{5} & \multirow{2}{*}{$\begin{array}{l}\text { SS1Ka SDP } \\
1-2 \mathrm{Ki}\end{array}$} & P.328 Right & 1.2 \\
\hline & & P.328 Left & 1.2 \\
\hline & \multicolumn{3}{|l|}{ SDP 1-2 Ki } \\
\hline \multirow[t]{2}{*}{6} & \multirow{2}{*}{$\begin{array}{l}\text { SS1Ka SDP } \\
2-2 \mathrm{Ka}\end{array}$} & P.313 A Right & 1.17 \\
\hline & & P.313 A Left & 1.17 \\
\hline \multirow[t]{2}{*}{7} & \multirow{2}{*}{$\begin{array}{l}\text { SS1Ki SDP } \\
2-2 \mathrm{Ka}\end{array}$} & P.311 A Left & 0.92 \\
\hline & & P.311 A Right & 0.92 \\
\hline 8 & $\begin{array}{l}\text { SS2Ka SDP } \\
2-2 \mathrm{Ka}\end{array}$ & BM PTG 10 & 1.18 \\
\hline \multirow[t]{2}{*}{9} & $\begin{array}{l}\text { SS2Ki SDP } \\
2-2 \mathrm{Ka}\end{array}$ & P.34 B & 1.14 \\
\hline & SDP 2-2 Ka & & \\
\hline \multirow[t]{2}{*}{10} & \multirow{2}{*}{$\begin{array}{l}\text { SS1Ka SDP } \\
2-2 \mathrm{Ki}\end{array}$} & P.361 A Right & 1.88 \\
\hline & & P.361 A Left & 1.88 \\
\hline \multirow[t]{3}{*}{11} & \multirow{2}{*}{$\begin{array}{l}\text { SS1Ki SDP } \\
2-2 \mathrm{Ki}\end{array}$} & P.343 Left & 1.58 \\
\hline & & P.343 Right & 1.58 \\
\hline & \multicolumn{3}{|l|}{ SDP $2-2 \mathrm{Ki}$} \\
\hline 12 & $\begin{array}{l}\text { SS1Ki SDP } \\
1-1 \mathrm{Ka}\end{array}$ & P.90 A & 1.79 \\
\hline \multirow[t]{3}{*}{13} & \multirow{2}{*}{$\begin{array}{l}\text { SS1Ki SDP } \\
1-1 \mathrm{Ka}\end{array}$} & P.366 Right & 1 \\
\hline & & P.366 Left & 1 \\
\hline & \multicolumn{3}{|l|}{ SDP 1-1 Ka } \\
\hline \multirow[t]{2}{*}{14} & \multirow{2}{*}{$\begin{array}{l}\text { SS1Ka SDP } \\
2-1 \mathrm{Ka}\end{array}$} & P.377 A Left & 1.83 \\
\hline & & P.377 A Right & 1.83 \\
\hline \multirow[t]{2}{*}{15} & \multirow{2}{*}{$\begin{array}{l}\text { SS1Ki SDP } \\
2-1 \mathrm{Ka}\end{array}$} & P.447 A Right & 2.34 \\
\hline & & P.447 A Left & 2.34 \\
\hline \multirow[t]{3}{*}{16} & \multirow{2}{*}{$\begin{array}{l}\text { SS2Ki SDP } \\
2-1 \mathrm{Ka}\end{array}$} & P.377 A Right & 1.83 \\
\hline & & P.377 A Left & 1.83 \\
\hline & SDP 2-1 Ka & & \\
\hline
\end{tabular}

\begin{tabular}{|c|c|c|c|}
\hline No & Channel & Stake Point & hw (m) \\
\hline \multirow[t]{2}{*}{17} & \multirow{2}{*}{$\begin{array}{l}\text { SS1Ka SDP } \\
3-1 \mathrm{Ka}\end{array}$} & P.441 A Right & 1.7 \\
\hline & & P.441 A Left & 1.7 \\
\hline \multirow[t]{2}{*}{18} & \multirow{2}{*}{$\begin{array}{l}\text { SS1Ki SDP } \\
3-1 \mathrm{Ka}\end{array}$} & P.439 A Left & 1.72 \\
\hline & & P.439 A Right & 1.72 \\
\hline \multirow[t]{2}{*}{19} & \multirow{2}{*}{$\begin{array}{l}\text { SS2Ka SDP } \\
3-1 \mathrm{Ka}\end{array}$} & P.385 Right & 1.22 \\
\hline & & P.385 Left & 1.22 \\
\hline \multirow[t]{2}{*}{20} & \multirow{2}{*}{$\begin{array}{l}\text { SS2Ki SDP } \\
3-1 \mathrm{Ka}\end{array}$} & P.385 Left & 1.22 \\
\hline & & P.385 Right & 1.22 \\
\hline 21 & $\begin{array}{l}\text { SS3Ka SDP } \\
3-1 \mathrm{Ka}\end{array}$ & P.354 A & 1.75 \\
\hline \multirow[t]{2}{*}{22} & $\begin{array}{l}\text { SS3Ki SDP } \\
3-1 \mathrm{Ka}\end{array}$ & P.354 A & 1.75 \\
\hline & SDP 3-1 Ka & & \\
\hline \multirow[t]{2}{*}{23} & \multirow{2}{*}{$\begin{array}{l}\text { SS1Ka SDP } \\
3-2 \mathrm{Ka}\end{array}$} & P.297 A Right & 1.52 \\
\hline & & P.297 A Left & 1.52 \\
\hline \multirow[t]{2}{*}{24} & $\begin{array}{l}\text { SS2Ka SDP } \\
3-2 \mathrm{Ka}\end{array}$ & P.43 A-1 & 1.5 \\
\hline & SDP $3-2 \mathrm{Ka}$ & & \\
\hline \multirow[t]{2}{*}{25} & \multirow{2}{*}{$\begin{array}{l}\text { SS1Ka SDP } \\
3-2 \mathrm{Ki}\end{array}$} & P.487 Right & 1.45 \\
\hline & & P.487 Left & 1.45 \\
\hline \multirow[t]{3}{*}{26} & \multirow{2}{*}{$\begin{array}{l}\text { SS1Ki SDP } \\
3-2 \mathrm{Ki}\end{array}$} & P.345 A Left & 1.21 \\
\hline & & P.345 A Right & 1.21 \\
\hline & \multicolumn{3}{|l|}{ SDP 3-2 Ki } \\
\hline \multirow[t]{2}{*}{27} & \multirow{2}{*}{$\begin{array}{l}\text { SS1Ka SDP } \\
4-1 \mathrm{Ka}\end{array}$} & P.428 A Right & 1.55 \\
\hline & & P.428 A Left & 1.55 \\
\hline \multirow[t]{2}{*}{28} & \multirow{2}{*}{$\begin{array}{l}\text { SS2Ka SDP } \\
4-1 \mathrm{Ka}\end{array}$} & P.396 Right & 1.3 \\
\hline & & P.396 Left & 1.3 \\
\hline \multirow[t]{2}{*}{29} & \multirow{2}{*}{$\begin{array}{l}\text { SS1Ki SDP } \\
4-1 \mathrm{Ka}\end{array}$} & A.18-A Left & 0.81 \\
\hline & & A.18-A Right & 0.81 \\
\hline \multirow[t]{2}{*}{30} & \multirow{2}{*}{$\begin{array}{l}\text { SS2Ki SDP } \\
4-1 \mathrm{Ka}\end{array}$} & P.426 A Left & 1.35 \\
\hline & & P.426 A Right & 1.35 \\
\hline \multirow[t]{3}{*}{31} & \multirow{2}{*}{$\begin{array}{l}\text { SS3Ki SDP } \\
4-1 \mathrm{Ka}\end{array}$} & P.396 Left & 1.3 \\
\hline & & P.396 Right & 1.3 \\
\hline & \multicolumn{3}{|l|}{ SDP 4-1 Ka } \\
\hline 32 & SS1Ka SDP & A.24-A Right & 0.78 \\
\hline & $5-1 \mathrm{Ka}$ & A.24-A Left & 0.78 \\
\hline 33 & SS1Ki SDP & A.27-A Left & 0.75 \\
\hline & $5-1 \mathrm{Ka}$ & A.27-A Right & 0.75 \\
\hline 34 & SS2Ka SDP & P.418 Right & 1.11 \\
\hline & $5-1 \mathrm{Ka}$ & P.418 Left & 1.11 \\
\hline 35 & & P.417 A Left & 1.37 \\
\hline
\end{tabular}




\begin{tabular}{clcc}
\hline No & Channel & Stake Point & hw (m) \\
\hline & SS2Ki SDP & P.417 A Right & 1.37 \\
& 5-1 Ka & & \\
\hline 36 & SS3Ka SDP & P.405 & 0.4 \\
& 5-1 Ka & & 0.4 \\
\hline 37 & SS3Ki SDP & P.405 & \\
& 5-1 Ka & & \\
\hline & SDP 5-1 Ka & & \\
\hline
\end{tabular}

\begin{tabular}{llcc}
\hline No & Channel & Stake Point & hw (m) \\
\hline $\mathbf{3 8}$ & SS1Ka SDP & A.32 Right & 0.65 \\
\cline { 3 - 4 } & 6-1Ka & A.32 Left & 0.65 \\
\hline $\mathbf{3 9}$ & $\begin{array}{l}\text { SS2Ka SDP } \\
\text { 6-1 Ka }\end{array}$ & CP.24 & 0.71 \\
\hline & SDP 6-1 Ka & & \\
\hline
\end{tabular}

The height of the canal water level profile (hw) that has been calculated on Table 1, are used to be one of the parameters at calculating ground water level by Ellipse Kirhkam method.

The groundwater level analysis was carried out using the modified Kirkham ellipse model method. In the calculation it is assumed that the elliptical model for the groundwater table intrudes the concept of mirror image (Figure 1).

With a value of $\mathrm{K}=180576 \mathrm{~mm} /$ day, the following is a recapitulation of the results of the groundwater level.

Table 2. Groundwater Level Recapitulation

\begin{tabular}{|c|c|c|c|}
\hline No & Channel & Stake Point & $h(x)(m)$ \\
\hline \multirow[t]{5}{*}{1} & \multirow{5}{*}{$\begin{array}{l}\text { S Drain Induk } \\
1\end{array}$} & P.216 B & 3.402 \\
\hline & & P. 243 B & 0.931 \\
\hline & & P. 253 & 0.823 \\
\hline & & P. 261 & 3.128 \\
\hline & & P. $92 \mathrm{~B}$ & 3.043 \\
\hline \multirow[t]{6}{*}{2} & \multirow{6}{*}{$\begin{array}{l}\text { S Drain Induk } \\
2\end{array}$} & P.131 B Right & 2.746 \\
\hline & & P.131 B Left & 2.755 \\
\hline & & P. 25 B Right & 3.477 \\
\hline & & P.25 B Left & 3.495 \\
\hline & & P. 11 A Right & 1.709 \\
\hline & & P. 11 A Left & 1.725 \\
\hline \multirow[t]{2}{*}{3} & \multirow{2}{*}{$\begin{array}{l}\text { SS1Ki SDP 1- } \\
2 \mathrm{Ka}\end{array}$} & P.325 A Left & 1.583 \\
\hline & & P.325 A Right & 1.592 \\
\hline 4 & $\begin{array}{l}\text { SS2Ki SDP 1- } \\
2 \mathrm{Ka} \\
\text { SDP 1-2 Ka }\end{array}$ & CP. 14 & 1.962 \\
\hline \multirow[t]{3}{*}{5} & \multirow{2}{*}{$\begin{array}{l}\text { SS1Ka SDP } \\
1-2 \mathrm{Ki}\end{array}$} & P.328 Right & 1.499 \\
\hline & & P.328 Left & 1.478 \\
\hline & SDP $1-2 \mathrm{Ki}$ & & \\
\hline \multirow[t]{2}{*}{6} & \multirow{2}{*}{$\begin{array}{l}\text { SS1Ka SDP } \\
2-2 \mathrm{Ka}\end{array}$} & P.313 A Right & 1.413 \\
\hline & & P.313 A Left & 1.403 \\
\hline \multirow[t]{2}{*}{7} & \multirow{2}{*}{$\begin{array}{l}\text { SS1Ki SDP 2- } \\
2 \mathrm{Ka}\end{array}$} & P.311 A Left & 0.886 \\
\hline & & P.311 A Right & 0.891 \\
\hline 8 & $\begin{array}{l}\text { SS2Ka SDP } \\
2-2 \mathrm{Ka}\end{array}$ & BM PTG 10 & 1.426 \\
\hline 9 & $\begin{array}{l}\text { SS2Ki SDP 2- } \\
2 \mathrm{Ka}\end{array}$ & P.34 B & 1.349 \\
\hline
\end{tabular}

\begin{tabular}{|c|c|c|c|}
\hline No & Channel & Stake Point & $h(x)(m)$ \\
\hline & SDP 2-2 Ka & & \\
\hline \multirow[t]{2}{*}{10} & \multirow{2}{*}{$\begin{array}{l}\text { SS1Ka SDP } \\
2-2 \mathrm{Ki}\end{array}$} & P.361 A Right & 3.608 \\
\hline & & P.361 A Left & 3.585 \\
\hline \multirow[t]{3}{*}{11} & \multirow{2}{*}{$\begin{array}{l}\text { SS1Ki SDP 2- } \\
2 \mathrm{Ki}\end{array}$} & P.343 Left & 2.564 \\
\hline & & P.343 Right & 2.543 \\
\hline & \multicolumn{3}{|l|}{ SDP 2-2 Ki } \\
\hline 12 & $\begin{array}{l}\text { SS1Ki SDP 1- } \\
1 \mathrm{Ka}\end{array}$ & P.90 A & 3.257 \\
\hline \multirow[t]{3}{*}{13} & \multirow{2}{*}{$\begin{array}{l}\text { SS1Ki SDP 1- } \\
1 \mathrm{Ka}\end{array}$} & P.366 Right & 1.035 \\
\hline & & P.366 Left & 1.039 \\
\hline & SDP 1-1 Ka & & \\
\hline \multirow[t]{2}{*}{14} & \multirow{2}{*}{$\begin{array}{l}\text { SS1Ka SDP } \\
2-1 \mathrm{Ka}\end{array}$} & P.377 A Left & 3.400 \\
\hline & & P.377 A Right & 3.417 \\
\hline \multirow[t]{2}{*}{15} & \multirow{2}{*}{$\begin{array}{l}\text { SS1Ki SDP 2- } \\
1 \mathrm{Ka}\end{array}$} & P.447 A Right & 5.540 \\
\hline & & P.447 A Left & 5.513 \\
\hline \multirow[t]{3}{*}{16} & \multirow{2}{*}{$\begin{array}{l}\text { SS2Ki SDP 2- } \\
1 \mathrm{Ka}\end{array}$} & P.377 A Right & 3.399 \\
\hline & & P.377 A Left & 3.406 \\
\hline & SDP 2-1 Ka & & \\
\hline \multirow[t]{2}{*}{17} & \multirow{2}{*}{$\begin{array}{l}\text { SS1Ka SDP } \\
3-1 \mathrm{Ka}\end{array}$} & P.441 A Right & 2.934 \\
\hline & & P.441 A Left & 2.943 \\
\hline \multirow[t]{2}{*}{18} & \multirow{2}{*}{$\begin{array}{l}\text { SS1Ki SDP 3- } \\
1 \mathrm{Ka}\end{array}$} & P.439 A Left & 3.003 \\
\hline & & P.439 A Right & 3.012 \\
\hline \multirow[t]{2}{*}{19} & \multirow{2}{*}{$\begin{array}{l}\text { SS2Ka SDP } \\
3-1 \mathrm{Ka}\end{array}$} & P.385 Right & 1.534 \\
\hline & & P.385 Left & 1.533 \\
\hline \multirow[t]{2}{*}{20} & \multirow{2}{*}{$\begin{array}{l}\text { SS2Ki SDP 3- } \\
1 \mathrm{Ka}\end{array}$} & P.385 Left & 1.534 \\
\hline & & P.385 Right & 1.533 \\
\hline
\end{tabular}




\begin{tabular}{|c|c|c|c|}
\hline No & Channel & Stake Point & $\mathbf{h}(\mathbf{x})(\mathbf{m})$ \\
\hline 21 & $\begin{array}{l}\text { SS3Ka SDP } \\
3-1 \mathrm{Ka}\end{array}$ & P.354 A & 3.116 \\
\hline \multirow[t]{2}{*}{22} & $\begin{array}{l}\text { SS3Ki SDP 3- } \\
1 \mathrm{Ka}\end{array}$ & P.354 A & 3.116 \\
\hline & SDP 3-1 Ka & & \\
\hline \multirow[t]{2}{*}{23} & \multirow{2}{*}{$\begin{array}{l}\text { SS1Ka SDP } \\
3-2 \mathrm{Ka}\end{array}$} & P.297 A Right & 2.365 \\
\hline & & P.297 A Left & 2.365 \\
\hline 24 & $\begin{array}{l}\text { SS2Ka SDP } \\
3-2 \mathrm{Ka} \\
\text { SDP 3-2 Ka }\end{array}$ & P.43 A-1 & 2.305 \\
\hline \multirow[t]{2}{*}{25} & \multirow{2}{*}{$\begin{array}{l}\text { SS1Ka SDP } \\
3-2 \mathrm{Ki}\end{array}$} & P.487 Right & 2.165 \\
\hline & & P. 487 Left & 2.155 \\
\hline \multirow[t]{3}{*}{26} & \multirow{2}{*}{$\begin{array}{l}\text { SS1Ki SDP 3- } \\
2 \mathrm{Ki}\end{array}$} & P.345 A Left & 1.521 \\
\hline & & P.345 A Right & 1.512 \\
\hline & SDP 3-2 Ki & & \\
\hline \multirow[t]{2}{*}{27} & \multirow{2}{*}{$\begin{array}{l}\text { SS1Ka SDP } \\
4-1 \mathrm{Ka}\end{array}$} & P.428 A Right & 2.461 \\
\hline & & P.428 A Left & 2.454 \\
\hline \multirow[t]{2}{*}{28} & \multirow{2}{*}{$\begin{array}{l}\text { SS2Ka SDP } \\
4-1 \mathrm{Ka}\end{array}$} & P.396 Right & 1.737 \\
\hline & & P.396 Left & 1.740 \\
\hline \multirow[t]{2}{*}{29} & \multirow{2}{*}{$\begin{array}{l}\text { SS1Ki SDP 4- } \\
1 \mathrm{Ka}\end{array}$} & A.18-A Left & 0.684 \\
\hline & & A.18-A Right & 0.685 \\
\hline \multirow[t]{2}{*}{30} & \multirow{2}{*}{$\begin{array}{l}\text { SS2Ki SDP 4- } \\
1 \mathrm{Ka}\end{array}$} & P.426 A Left & 1.860 \\
\hline & & P.426 A Right & 1.871 \\
\hline 31 & & P.396 Left & 1.737 \\
\hline
\end{tabular}

\begin{tabular}{|c|c|c|c|}
\hline No & Channel & Stake Point & $\mathbf{h}(\mathbf{x})(\mathbf{m})$ \\
\hline & $\begin{array}{l}\text { SS3Ki SDP 4- } \\
1 \mathrm{Ka}\end{array}$ & P.396 Right & 1.740 \\
\hline 32 & $\begin{array}{c}\text { SS1Ka SDP } \\
5-1 \mathrm{Ka}\end{array}$ & A.24-A Right & 0.644 \\
\hline \multirow{2}{*}{$\begin{array}{l}32 \\
33\end{array}$} & \multirow{2}{*}{$\begin{array}{l}\text { SS1Ka SDP } \\
5-1 \mathrm{Ka} \\
\text { SS1Ki SDP 5- } \\
1 \mathrm{Ka}\end{array}$} & A.24-A Left & 0.637 \\
\hline & & A.27-A Left & 0.597 \\
\hline \multirow{2}{*}{$\begin{array}{l}33 \\
34\end{array}$} & \multirow{2}{*}{$\begin{array}{l}\text { SS1Ki SDP 5- } \\
1 \mathrm{Ka} \\
\text { SS2Ka SDP } \\
5-1 \mathrm{Ka}\end{array}$} & A.27-A Right & 0.591 \\
\hline & & P.418 Right & 1.267 \\
\hline \multirow{2}{*}{$\begin{array}{l}34 \\
35\end{array}$} & \multirow{2}{*}{$\begin{array}{l}\text { SS2Ka SDP } \\
5-1 \mathrm{Ka} \\
\text { SS2Ki SDP 5- } \\
1 \mathrm{Ka}\end{array}$} & P.418 Left & 1.276 \\
\hline & & P.417 A Left & 1.915 \\
\hline \multirow{2}{*}{$\begin{array}{l}35 \\
36\end{array}$} & \multirow{2}{*}{$\begin{array}{l}\text { SS2Ki SDP 5- } \\
1 \mathrm{Ka} \\
\text { SS3Ka SDP } \\
5-1 \mathrm{Ka}\end{array}$} & P.417 A Right & 1.926 \\
\hline & & P.405 & 0.186 \\
\hline \multirow[t]{2}{*}{37} & $\begin{array}{l}\text { SS3Ki SDP 5- } \\
1 \mathrm{Ka}\end{array}$ & P.405 & 0.186 \\
\hline & SDP 5-1 Ka & & \\
\hline 38 & $\begin{array}{l}\text { SS1Ka SDP } \\
6-1 \mathrm{Ka}\end{array}$ & A.32 Right & 0.460 \\
\hline \multirow{3}{*}{$\begin{array}{l}38 \\
39\end{array}$} & \multirow{2}{*}{$\begin{array}{l}\text { SS1Ka SDP } \\
6-1 \mathrm{Ka} \\
\text { SS2Ka SDP } \\
6-1 \mathrm{Ka}\end{array}$} & A.32 Left & 0.448 \\
\hline & & CP. 24 & 0.531 \\
\hline & SDP 6-1 Ka & & \\
\hline
\end{tabular}

The table above is calculating the groundwater level $(\mathrm{hx})$ each stakes of the channel. The calculation is using the modified Kirkham elliptical model along with other parameters, namely evapotranspiration, effective rainfall, and soil conductivity. After obtaining the groundwater level at each stake point that calculated in Table 2, the groundwater level profile is drawn. The description of the groundwater table is carried out to be able to estimate whether the land is over-drained or not.

Land is said to be over drained if the groundwater level is $>70 \mathrm{~cm}$ below the land.

From the results of the analysis, it was found that over drained occurs a lot in the upstream part, namely in the primary channel at Main Canal I peg P.253 and P.243 B, Secondary Channel 2 Right and Left SDP 3-1 Ka, Secondary Channel 1 Left SDP 4 -1 Ka, Secondary Channel 1 Right and Left SDP 5$1 \mathrm{Ka}$, Secondary Channel 3 Right and Left SDP 5-1 Ka Secondary Channel 1 and 2 Right SDP 6-1 Ka. As for Main Channel II, only one channel is overdrained, namely Secondary Channel 1 Left SDP 2-2 Ka.

The following is an example of depicting a groundwater table to get the status of whether the land is over drained or not. 


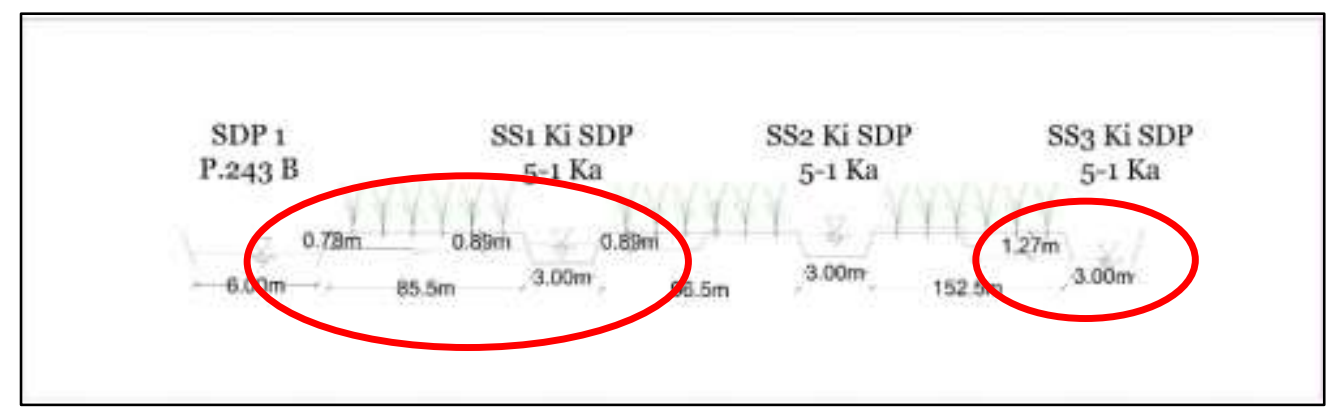

Figure 5. Example of Groundwater Profile Modeling Over Drained Condition

From Figure 5, there is an example of cross section that shows the groundwater level is not up to the standard $(<70 \mathrm{~cm})$ below the surface. Therefore, the land in SDP 1 P.243 B, SS1 Ki SDP 5-1 Ka, and SS3 Ki SDP 5-1 Ka experiencing over drain.

\section{Conclusion}

From the analysis carried out by depicting hydrotopographic maps, it is known that the study locations, namely Main Canal I and II of Petung Swamp Irrigation Area fall into the category of transitional lowland swamp. This means that the channel is still affected by the tides. In addition, from the analysis using hec-ras, it was found that the influence of the tides only reaches along the main channel. So, it can be said that the results of the hec-ras analysis are in accordance with the depiction of the hydrotopographic map.

From the survey results in the field, it can be seen that the dimensions of the channel in the Petung swamp drainage channel are more than adequate to accommodate the discharge that passes through the channel. However, there are no sluice gates at every intersection or meeting point of water which causes water regulation in the Petung swamp irrigation area to cause problems on agricultural land. In addition, water sources for irrigation of swamps, which come from tides and rainfall, cannot be used effectively, especially during the dry season which is prone to water shortages and can cause over-drained problems in swamps. From the analysis, it can be seen that some parts of agricultural land are over drained. Namely on the primary channel to the secondary channel on the main channel I and the secondary channel SS 1 Ki SDP 3-2 Ka on the main channel II. Thus, it can be classified the types of overflow in the Rawa Petung Irrigation Area, namely, type C overflow in Main Drainage Channels in Mains I and II, and Primary Channels in Main Canal II. Meanwhile, the Primary Channel in Main Canal I and all Secondary Channels in Petung Swamp Irrigation Area are overflow type D because they are not affected by tides.

The solution that can be done to overcome the problem of over-draining in petung swamp land is to build an automatic sluice gate (flapgate) at the channel crossing point along the main channel II so that the door can open automatically when there is high tide and close automatically when the water is low. In addition, it is also necessary to add a stoplog at every intersection from the primary to the secondary channel so that the interpreter can regulate the water and maintain the stability of the groundwater level. It is also necessary to build a reservoir in the secondary channel to accommodate rainwater in the rainy season and can be used in the dry season if there is a shortage of water.

With the problem channel mapped, the treatment can be carried out effectively. If the handling can be done properly and evenly, then the government's program of achieving the national food security can be achieved.

\section{Reference}

[1] Panggabean, E. W., \& Wiryawan, B. A. (2016). Strategy for Development of Swamp Irrigation Land in the Tidal Swamp Area of Belawang - South Kalimantan. Journal of Irrigation, Vol. 11 No. 1, 1-10. 
[2] Ar-Riza, \& Alkasuma. (2008). Tidal Swamp Farming and Its Development Strategy in the Era of Regional Autonomy. Journal of Land Resources, Vol. 2 No. 2, 95-104.

[3] Imanudin, M., Bakri, Armanto, E., Indra, B., \& Ratmini, S. (2018). Land and Water Management Option of Tidal Lowland Reclamation Area to Support Rice Production. Journal of Wetlands Environmental Management, Vol. 6 No. 2, 93-109.

[4] Mariana, Z. T., Mahbub, M., \& Hayati, A.(2015). Sustainable Land Management Support Organic Farming in Tidal Swamp Area of South Kalimantan: Water Quality. Journal of Wetlands Environmental Management, Vol 3 No. 2, 95-100.

[5] Pandjaitan, N. H., \& Hardjoamidjojo, S. (1999). Study of Physical Properties of Peatlands in Relationship with Drainage for Agricultural Land. Agricultural Engineering Bulletin, Vol. 13 No. 03, 87-96.

[6] Agus, F., Anda, M., Jamil, A., \& Masganti. (2014). Indonesian Peatlands. Bogor: IAARD Press.

[7] Herawati, H., Yulianto, E., \& Azmeri. (2020). The Effect of Hydrotopography and Land Use on Tertiary Channels in the Rawa Pinang Dalam Area. Scientific Journal, Vol. 20 No. 1, 1-10.

[8] Ngudiantoro, Pawitan, H., Ardiansyah, M., Purwanto, M. Y., \& Susanto, R. H. (2010). Modeling of Groundwater Level Fluctuations in Tidal Swamp Type B/C: Case in South Sumatra. Postgraduate Forum. Vol. 33 No. 2, 101-112.

[9] Dariah, A., Maftuah, E., \& Maswar. (2016). Peatland Characteristics. Guidelines for the Sustainable Management of Degraded Peatlands, 16-29.

[10] Haribowo R., Andawayanti U., Lufira R.D. 2019. Effectivity test of an eco-friendly sediment trap model as a strategy to control erosion on agricultural land. Journal of Water and Land Development. No. 42 (VII-IX) p. 76-82. DOI: 10.2478/jwld-2019-0047.

[11] Permata, V. Y., Siswoyo, H., \& Haribowo, R. (2021). Mapping of Groundwater Flow Pattern and its Quality Index based on Microbiological Parameters in Klojen District, Malang City, East Java, Indonesia.Civil and Environmental Science Journal (Civense), 4(2), 106-114. doi:https://doi.org/10.21776/ub.civense.2021.00402.1 